Artículo de investigación científica y tecnológica

Cómo citar: E. Alayón. “Guía para la caracterización y cuantificación de residuos sólidos". Inventum, vol. 15 , no. 29 pp. 76-94. doi: 10.26620/uniminuto.inventum. 15.29.2020.76-94

Editorial: Corporación Universitaria Minuto de Dios - UNIMINUTO.

ISSN: $1909-2520$

eISSN: $2590-8219$

Fecha de recibido: 31 de mayo de 2020 Fecha de aprobado: 15 de junio de 2020 Fecha de publicación: 15 de julio de 2020

Conflicto de intereses: los autores han declarado que no existen intereses en competencia.

\section{Guía para la caracterización y cuantificación de residuos sólidos}

\author{
Guide for the characterization and \\ quantification of solid waste
}

\begin{abstract}
Resumen
La caracterización de los residuos sólidos es una etapa básica e importante dentro de su propia gestión, ya que busca identificar fuentes, cantidades y variaciones en el tiempo, al igual que la observación y calidad de los productos. El problema principal es que existen muy pocos métodos, que se emplean unicamente para caracterizar residuos sólidos municipales y urbanos, situación que lo hace aún más complejo. Por lo anterior, se propone esta guía que presenta un método para caracterizar y cuantificar de una manera más sencilla y eficaz, no solo los residuos sólidos municipales y urbanos, sino también los institucionales, comerciales e incluso de fincas, es decir conocer los componentes individuales que constituyen el flujo de los residuos, usualmente basados en porcentaje por peso.
\end{abstract}

Una vez conocida la distribución de los componentes se procede a cuantificarlos con el fin de obtener la producción por persona, también llamada producción per cápita ( $p p c)$, cuyas unidades son $\mathrm{Kg}$ / hb.día. La producción per cápita es estratificada especialmente en América, de tal manera que entre más alto sea el estrato socioeconómico de un país, municipio o barrio, mayor es la generación de residuos. En Colombia las ciudades capitales presentan 6 estratos, y los municipios generalmente 3 , siendo el 1 el más bajo.

Se concluye que, para el método cuantitativo presentado en esta guía, cuando el resultado de la ppc obtenida por cálculos coincide con la ppc del estrato real en donde se esté trabajando, confirma que la cuantificación estuvo bien realizada, y por consiguiente la caracterización también es correcta.

Palabras clave: caracterización de residuos, composición de residuos, generación de residuos, producción per cápita, residuos sólidos.

\begin{abstract}
The characterization of solid waste is an important basic stage in the management of solid waste, and important within its own management, since it seeks to identify sources, quantities and variations over time as well as the observation and quality of the products. The problem is there
\end{abstract}


are a few methods only to characterize municipal and urban solid waste, circumstance that is even more complex. Therefore, it is proposed that this guide presents a method to characterize and quantify in a simpler and more effective way not only municipal and urban solid waste, but also institutional, commercial and even farm waste, i.e. to know the individual components that constitute the flow of waste, usually based on percentage by weight.

Once the distribution of the components is known, they are quantified in order to obtain the production per person, also called per capita production (ppc) whose units are $\mathrm{Kg} / \mathrm{hb}$.day. Per capita production is particularly stratified in America, so that the higher the socioeconomic stratum of a country, municipality or neighborhood, the greater the waste generation. In Colombia, the capital cities have 6 strata, and the municipalities generally 3 , being the 1 the lowest.

It is concluded that for the quantitative method presented in this guide, when the result of the ppc obtained by calculations coincides with the ppc of the actual stratum in which the work is being worked, it confirms that the quantification was well realized and therefore the characterization is also correct.

Keywords: Waste characterization, waste composition, waste generation, per capita production, solid waste.

\section{Resumo}

A caracterização dos resíduos sólidos é uma etapa básica e importante dentro da sua própria gestão, uma vez que busca identificar fontes, quantidades e variações ao longo do tempo, bem como a observação e qualidade dos produtos. O principal problema é que existem poucos métodos, utilizados apenas para caracterizar os resíduos sólidos urbanos e municipais, situação que a torna ainda mais complexa. Portanto, propõe-se este guia, que apresenta um método para caracterizar e quantificar de forma mais simples e eficiente, não só resíduos sólidos urbanos e urbanos, mas também resíduos institucionais, comerciais e até agrícolas, ou seja, conhecer os componentes individuais que compõem o fluxo de resíduos, geralmente com base na porcentagem por peso.

Uma vez conhecida a distribuição dos componentes, eles são quantificados para se obter a produção por pessoa, também chamada de produção per capita (ppc) cujas unidades são Kg / hb.dia. A produção per capita é particularmente estratificada na América, de modo que quanto maior o estrato socioeconômico de um país, município ou bairro, maior a geração de resíduos. Na Colômbia, as capitais apresentam 6 estratos e os municípios geralmente 3 , sendo 1 o mais baixo.

Conclui-se que para o método quantitativo apresentado neste guia, quando o resultado do ppc obtido por cálculos coincide com o ppc do estrato real em que o trabalho está sendo realizado, confirma que a quantificação foi bem realizada e, portanto, a caracterização também está correta.

Palavras-chave: Caracterização de residuos, composição de residuos, geração de residuos, produção per capita, resíduos sólidos. 


\section{I.INTRODUCCIÓN}

El problema de la evacuación de los residuos sólidos inicia desde que los seres humanos comenzaron a agruparse en tribus, aldeas y comunidades, de tal forma que su acumulación llegó a ser una consecuencia de la vida. Como producto de las actividades de una sociedad que incluye costumbres, capacidad adquisitiva y hábitos diarios.

Las condiciones geográficas y económicas de un país, municipio o ciudad, la complejidad y efectividad en el manejo de los residuos sólidos, han hecho que sea imprescindible un mayor conocimiento de la composición y generación para realizar una buena disposición de los residuos.

Para determinar la composición de los residuos sólidos es necesario realizar una caracterización, que consiste en determinar la composición de los residuos en sus diferentes fracciones.

La composición de los residuos sólidos [1], es el término usado para describir los componentes individuales que constituyen el flujo de los residuos y su distribución relativa, usualmente basada en porcentaje por peso.

Las caracterizaciones detalladas de residuos sólidos son cruciales para identificar el potencial de reciclaje, de tratamiento y las opciones de disposición final, lo cual necesita ser adaptado a las condiciones locales para cada caso [2], lo mismo que para evaluar las necesidades de equipo y personal. Por ello, las tecnologías seleccionadas dependen de la cantidad y la composición de los residuos, que además permiten estimar los impactos potenciales en el medio ambiente asociados con la disposición inadecuada de los residuos sólidos.

En la actualidad existen muy pocos métodos para llevar a cabo una caracterización, y los que hay son muy difíciles de implementar, como el presentado por la OPS/CEPIS/04/IT-634 [3], que prácticamente es el mismo en [4] y la Norma Mexicana NMX- AA015 - 1985 [5], forman parte de los antecedentes para plantear esta investigación.

Difíciles de implementar, porque toman grandes cantidades de muestra, mencionan alrededor de 250 bolsas o sea como mínimo $2000 \mathrm{Kg}$, que luego reducen a $50 \mathrm{~kg}$ mediante la realización de tres o cuatro cuarteos, porque en cada uno de ellos se pierde la mitad de la muestra y la otra mitad la emplean en los siguientes cuarteos. Este método propuesto es importante por lo sencillo y eficaz, ya que solo utiliza de 13 a 15 bolsas, porque solo requiere $90 \mathrm{Kg}$ y se realiza con solo dos cuarteos. Además, que no es necesario trozar los residuos a tamaños manipulables $(15 \mathrm{~cm})$, como lo sugiere el Centro Panamericano de Ingeniería Sanitaria y Ciencias del Ambiente (Cepis), [3, p. 69], sino que se caracterizan tal como se encuentran.

Por la naturaleza heterogénea de los residuos sólidos, la determinación de la composición no es tarea fácil. Los procedimientos estadísticos estrictos son difíciles, por esto hay procedimientos de campo generalizados, basados en el sentido común y las técnicas de muestreo al azar.

Con el trabajo y la experiencia durante más de 17 años en el manejo y disposición de los residuos sólidos, se presenta esta investigación realizada en Colombia en dos municipios del departamento de Cundinamarca (Anolaima y Zipacón), en 50 barrios, 10 empresas y 2 universidades de Bogotá, con el objetivo de dar a conocer un método sencillo para caracterizar y cuantificar los residuos sólidos municipales, urbanos, institucionales y comerciales.

Durante los años 2004 y 2005 se realizaron varias caracterizaciones en dos barrios de Bogotá y en una universidad, se compararon las dos metodologías, la propuesta por el Cepis y la presentada en esta guía, con la aplicación de un método cuantitativo para la comparación. La diferencia fue de un $10 \%$, de tal manera que a partir del año 2005 las caracterizaciones en los demás barrios, universidades y empresas mencionadas anteriormente se llevaron a cabo con el método propuesto en esta guía.

\section{METODOLOGÍA PARA LA REALIZACIÓN DEL CUARTEO}

El método de cuarteo presentado en esta guía permite caracterizar diferentes sectores como el municipal, urbano, institucional, entre otros.

El método es el mismo, lo que cambia es la forma de realizar el muestreo de acuerdo con el origen de los residuos sólidos.

En Colombia los residuos urbanos (barrios), están conformados por residuos domésticos y comerciales. En otras palabras, cualquier barrio de nuestros municipios o ciudades tiene en su jurisdicción bastantes sitios comerciales dentro de las casas o en locales de edificios residenciales; teniendo en 
cuenta que actualmente nuestros Planes de Ordenamiento Territorial (POT) o los Planes Básicos de Ordenamiento Territorial (PBOT), no separan claramente estos dos sectores. Por lo tanto, para este sector, los residuos domésticos y comerciales prácticamente se caracterizan juntos, a excepción de los centros comerciales que se caracterizan como sector comercial, porque además la recolección de residuos la realiza un vehículo recolector distinto.

Si el origen es urbano, las muestras o bolsas de residuos se toman al azar en diferentes sitios del barrio, pero teniendo en cuenta que estén incluidos los sectores domésticos y comercial de la zona residencial para que la muestra sea homogénea.

En el caso de residuos municipales la muestra se toma directamente del camión de recolección sin ningún tipo de separación previa, y para que sea homogénea se palea de diferentes puntos del mismo.

Por consiguiente, para realizar el muestreo en un municipio o barrio, se hace necesario conocer los límites correspondientes y todas las características socioeconómicas de estos, así como el estrato del municipio o estrato del barrio al que pertenece dentro de la ciudad o municipio, población y producción de residuos sólidos.

Para el sector institucional, teniendo en cuenta que los residuos sólidos tienen una dinámica distinta ya que se debe maximizar la posibilidad de reducción, recuperación y aprovechamiento, es importante conocer la producción semanal y la población (estudiantes, docentes y administrativos), la muestra de los residuos sólidos se toma en forma representativa del centro de acopio o del sitio de almacenamiento de que se disponga.

Finalmente, para los sectores de origen comercial o en fincas, la muestra se toma directamente en el origen de la producción por día durante una semana y no de una carga mezclada de residuos de un vehículo de recogida.

Una vez elegidas, de acuerdo con el origen, las muestras de los residuos sólidos que se van a caracterizar y cuantificar, es decir elegido el municipio, barrio, el colegio, la universidad, la finca, entre otros; se ubica dentro de los sitios mencionados, un lugar amplio y despejado, aislado del viento y que no incomode a los transeúntes, vecinos y demás personas cercanas al espacio seleccionado.

En [1, p. 67], se ha encontrado que las medidas tomadas a partir de un muestreo del tamaño de unos
$90 \mathrm{Kg}$, no varían significativamente de las tomadas en muestreos de hasta $770 \mathrm{Kg}$ sacados de la misma carga de residuos.

Por lo tanto, con una carga de $90 \mathrm{Kg}$ se procede a realizar el cuarteo en 10 pasos, de la siguiente manera:

A manera de ejemplo se describirá cada uno de los pasos, con una caracterización llevada a cabo en el sector institucional, en este caso en la Universidad El Bosque.

1) Se toma la muestra de residuos del sitio de almacenamiento de la Universidad, figuras 1 y 2 , y se descarga directamente sobre el suelo, preferiblemente en un espacio pavimentado.

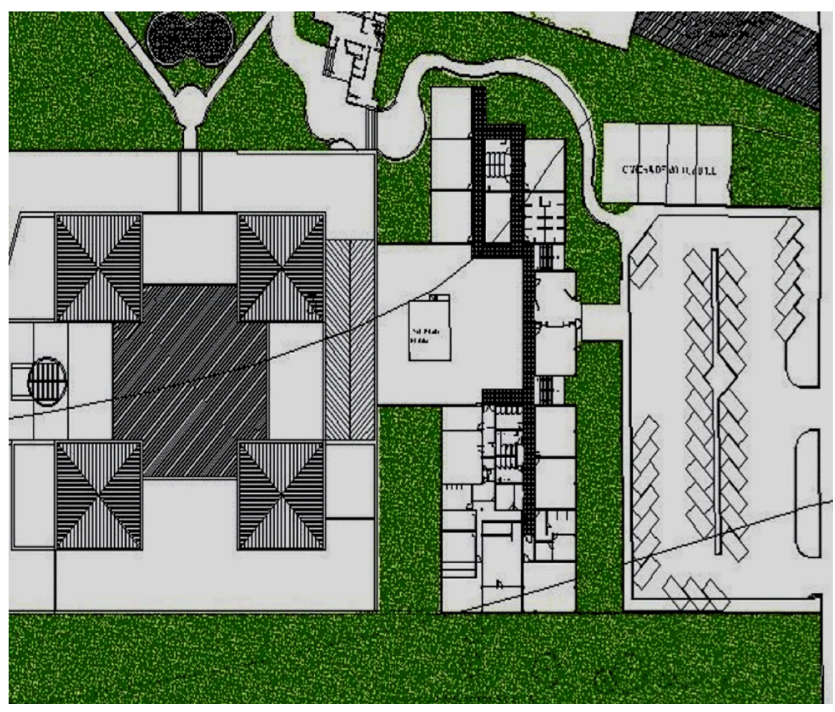

Figura 1. Plano de la Universidad.

Fuente: elaboración propia.

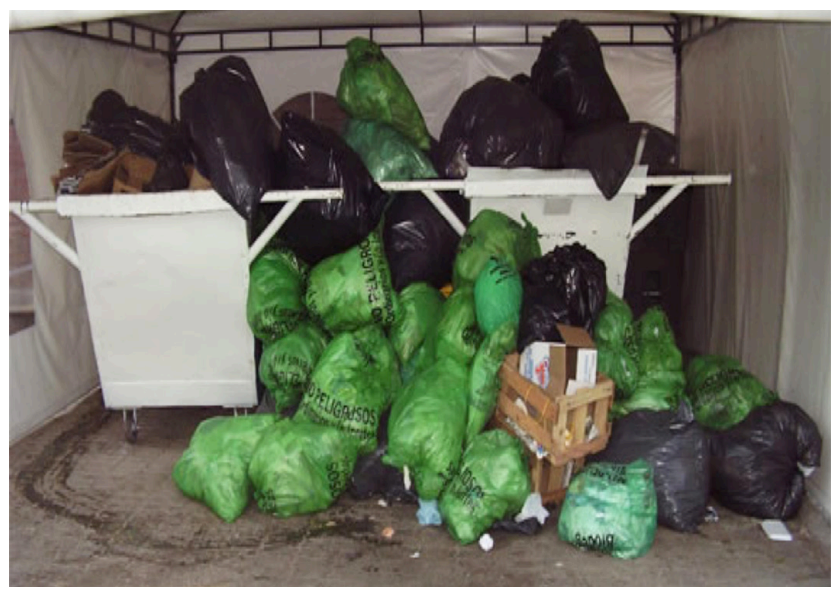

Figura 2. Sitio de almacenamiento de residuos sólidos. Fuente: elaboración propia. 
2) Se crea un círculo de aproximadamente $1.5 \mathrm{~m}$ de radio, y se subdivide en cuatro cuadrantes de igual tamaño, con su respectiva numeración I, II, III, IV, figuras 3 y 4 . Para que fueran más visibles las divisiones se colocaron ladrillos, pero en realidad solo se dibujan las líneas o son simplemente imaginarias.

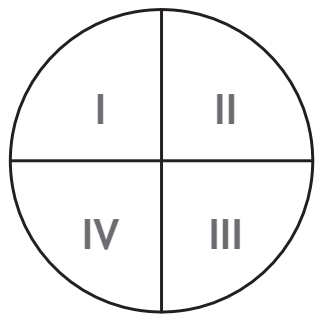

Figura 3. Cuarteo de residuos sólidos.

Fuente: elaboración propia.
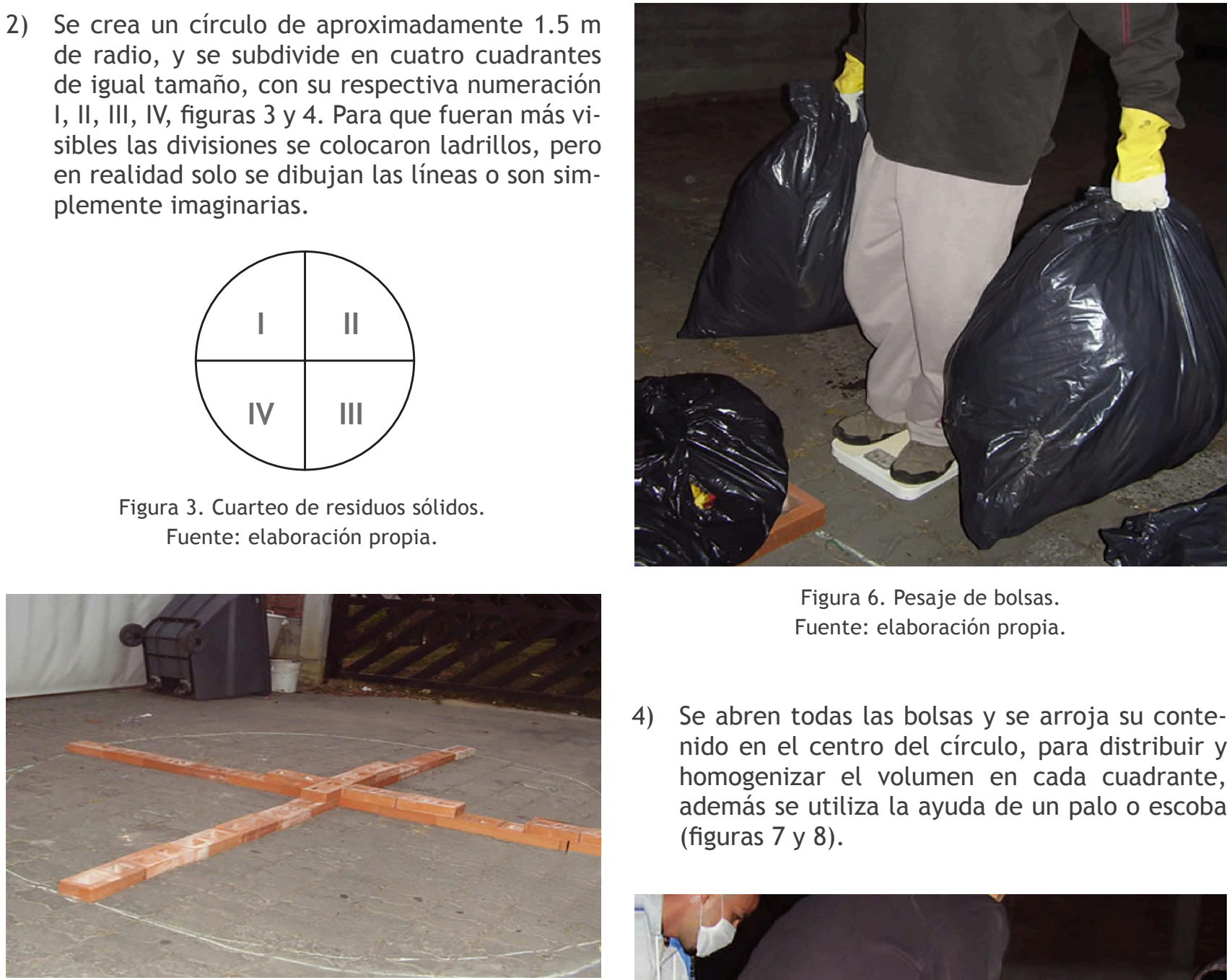

Figura 6. Pesaje de bolsas.

Fuente: elaboración propia.

Figura 4. Cuadrantes.

Fuente: elaboración propia.

3) Se hace la selección de bolsas de residuos y se pesan hasta que se alcance un peso total de 90 Kg (figuras 5 y 6 ).

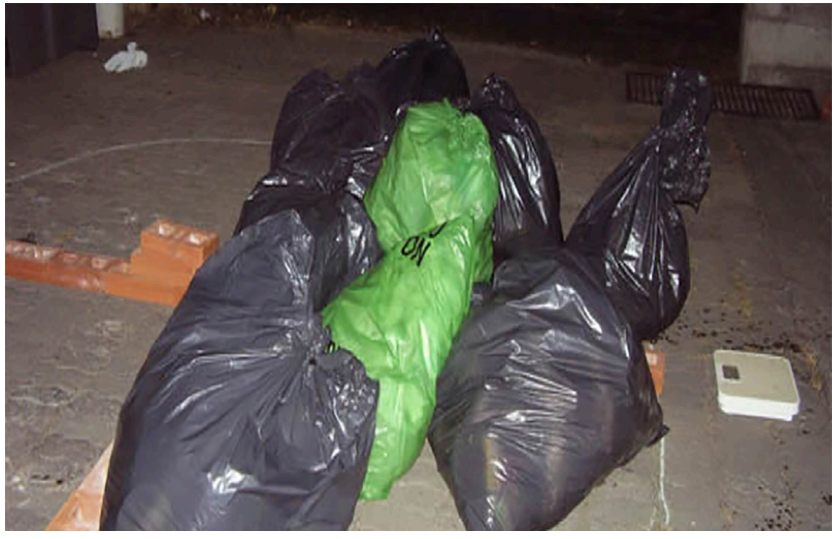

Figura 5. Selección de bolsas. Fuente: elaboración propia.

4) Se abren todas las bolsas y se arroja su contenido en el centro del círculo, para distribuir y homogenizar el volumen en cada cuadrante, además se utiliza la ayuda de un palo o escoba (figuras 7 y 8 ).

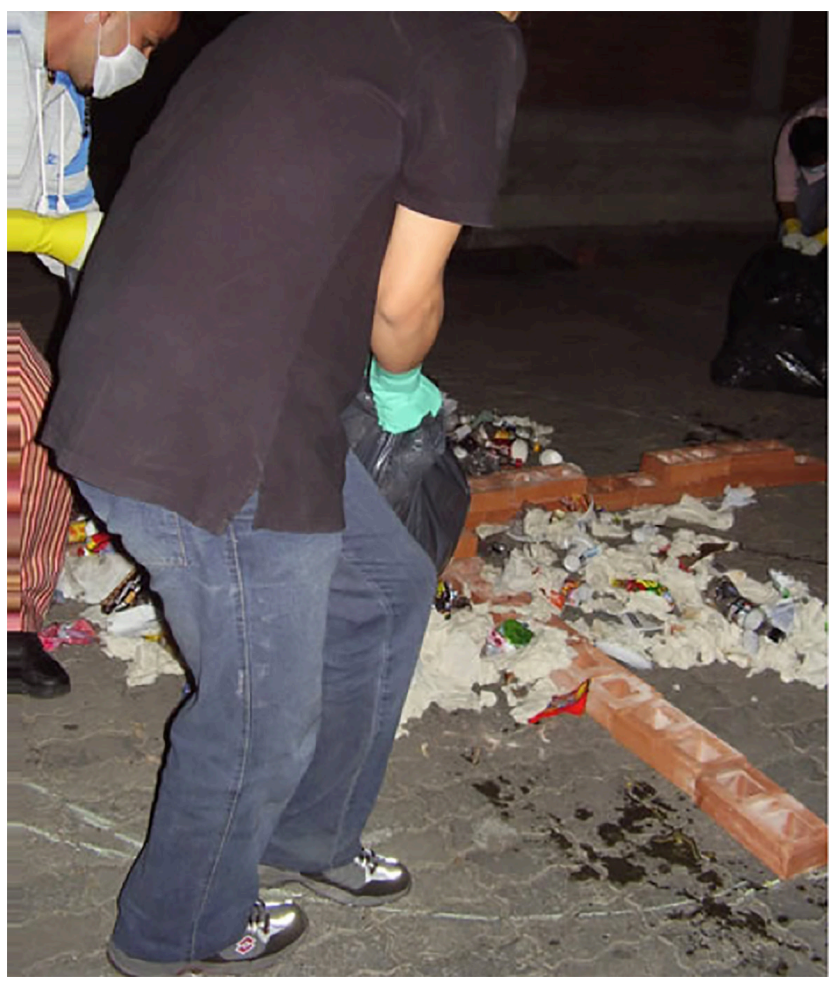

Figura 7. Residuos arrojados en el centro. Fuente: elaboración propia. 


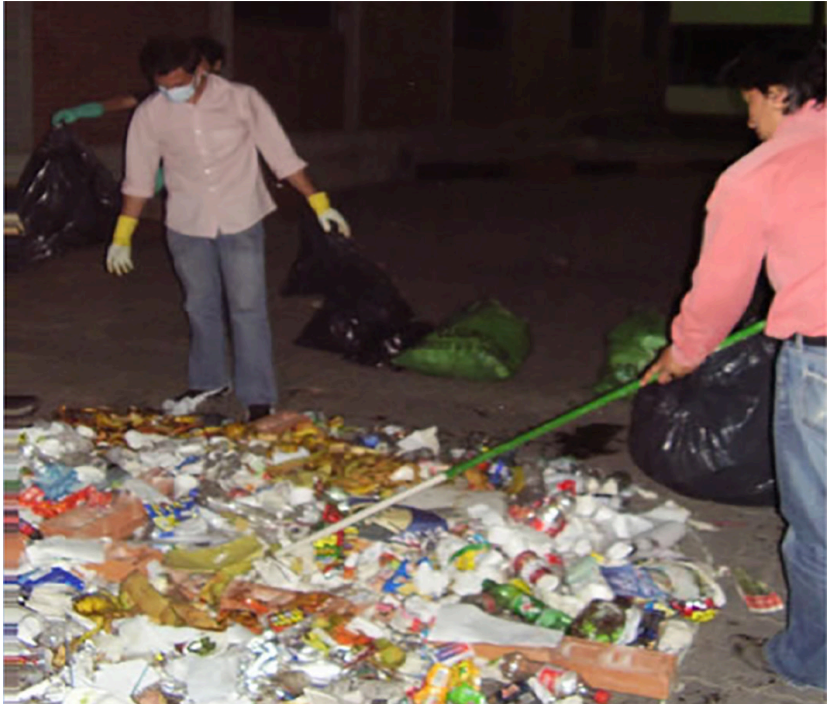

Figura 8. Homogenización de los residuos. Fuente: elaboración propia.

5) Se escogen 2 cuadrantes opuestos, estos son el I y el III; de esta forma se seleccionan los residuos sólidos por tipo de material en diferentes bolsas, así: vidrio, plástico, papel, cartón, textiles si se encuentran en cantidad considerable, latas de hojalata y aluminio (figura 9). Se recoge en la última bolsa el material putrescible que queda, como tierra de barrido llamada también suciedad, papel higiénico, cascaras, etc.

Es importante mantener la integridad de cada cuadrante seleccionado, independiente del olor o la descomposición física, para asegurar que todos los componentes son medidos. Solamente de esta manera se puede mantener algún grado de azar y una selección imparcial.

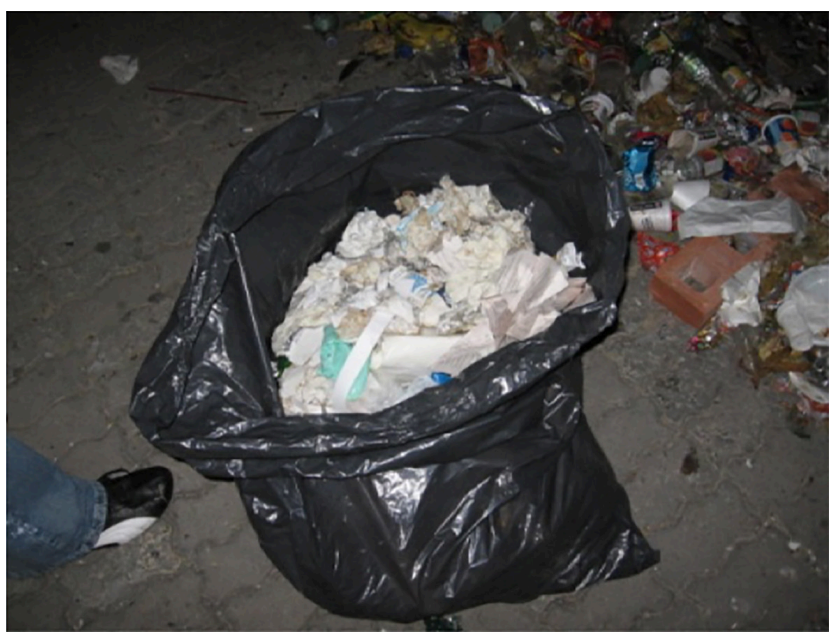

Figura 9. Selección de los residuos. Fuente: elaboración propia.
6) Se hace el pesaje de las bolsas por material separado, y se comparan los resultados de la suma del cuadrante I con el III, para determinar su semejanza en composición (figuras 10 y 11). Entre la sumatorias de los 2 cuadrantes solo puede haber una diferencia máxima de $2 \mathrm{Kg}$. Si esto se cumple significa que hubo una buena homogenización, se recogen todos los cuadrantes y se procede a calcular el promedio de los cuadrantes trabajados I y III.

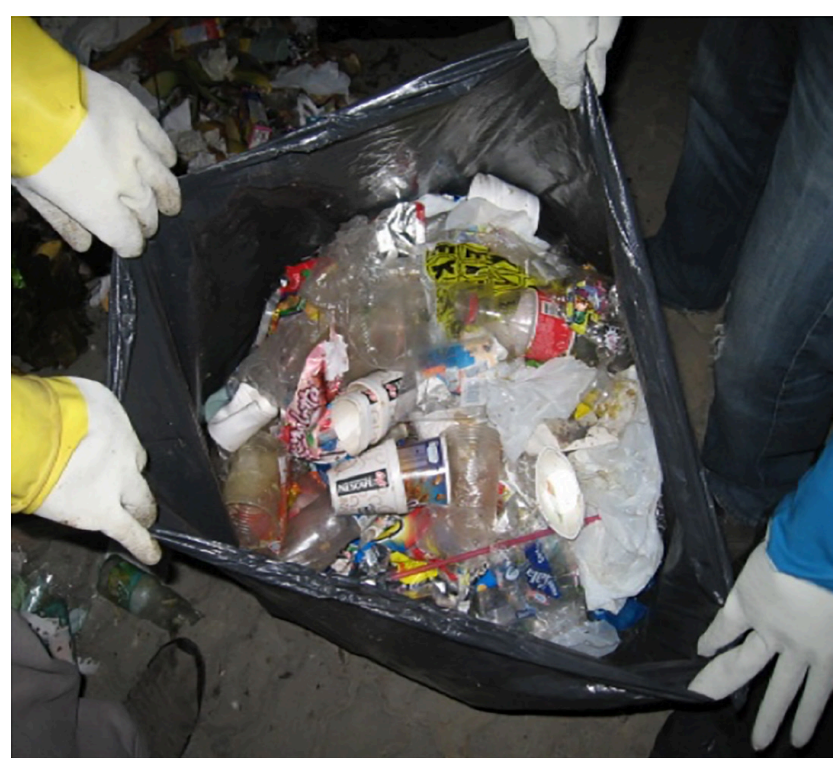

Figura 10. Cuadrante I.

Fuente: elaboración propia.

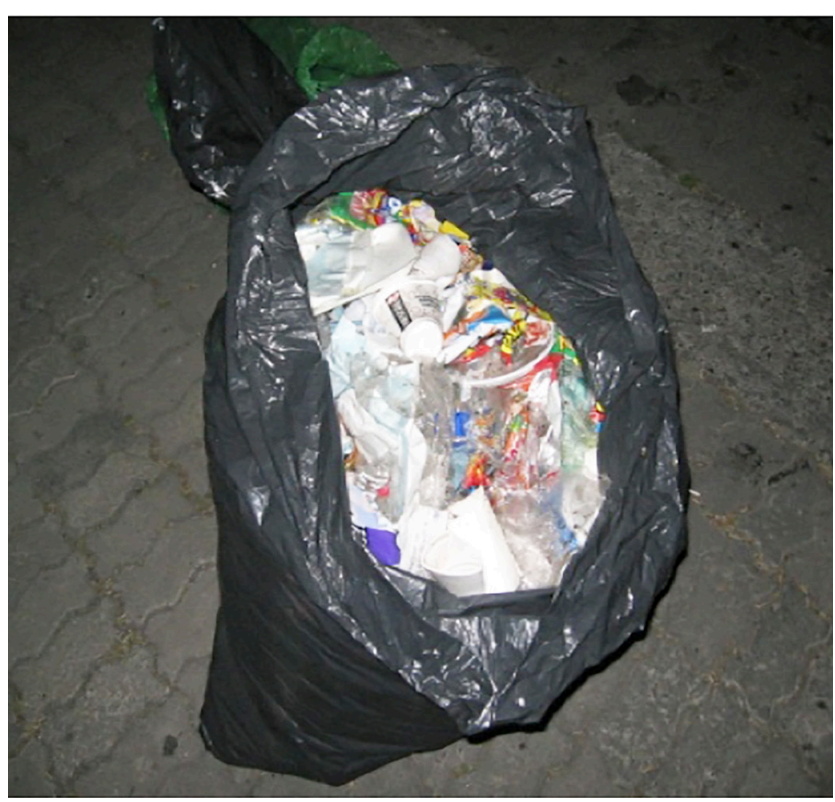

Figura 11. Cuadrante III.

Fuente: elaboración propia. 
7) Si, por el contrario, la composición de estos dos cuadrantes I y III es mayor a $2 \mathrm{Kg}$, se saca a un lado el cuadrante II y se repite el proceso de pesaje por bolsas para este cuadrante II. La sumatoria se puede comparar con la del cuadrante I o III, y se verifica que la diferencia sea menor a $2 \mathrm{~kg}$ con cualquiera de los dos cuadrantes para promediar. Ahora, si la sumatoria de los tres cuadrantes entre sí es menor de los $2 \mathrm{Kg}$ se promedia con los tres cuadrantes.

8) Si entre la sumatoria de los cuadrantes I, II y III la diferencia está por encima de $2 \mathrm{~kg}$, se hace necesario sacar a un lado el cuadrante IV y se repite el proceso de pesaje por bolsas para este cuadrante IV. La sumatoria de este último cuadrante se pude comparar con la sumatoria de cualquiera de los otros tres, para encontrar con cuál de ellos la diferencia es menor de $2 \mathrm{Kg}$ para poder promediar.

Si, aun así, con estas oportunidades de comparación entre cuadrantes la diferencia no es menor de $2 \mathrm{Kg}$, significa que el procedimiento no tuvo previamente una correcta homogenización.

9) Con los valores obtenidos de cada cuadrante se determina el promedio en peso y el porcentaje.

10) Lo último que se hace es recoger todos los residuos que estén en el círculo, se barre, para dejar el sitio limpio tal y como fue encontrado (figuras 12,13 y 14 ).

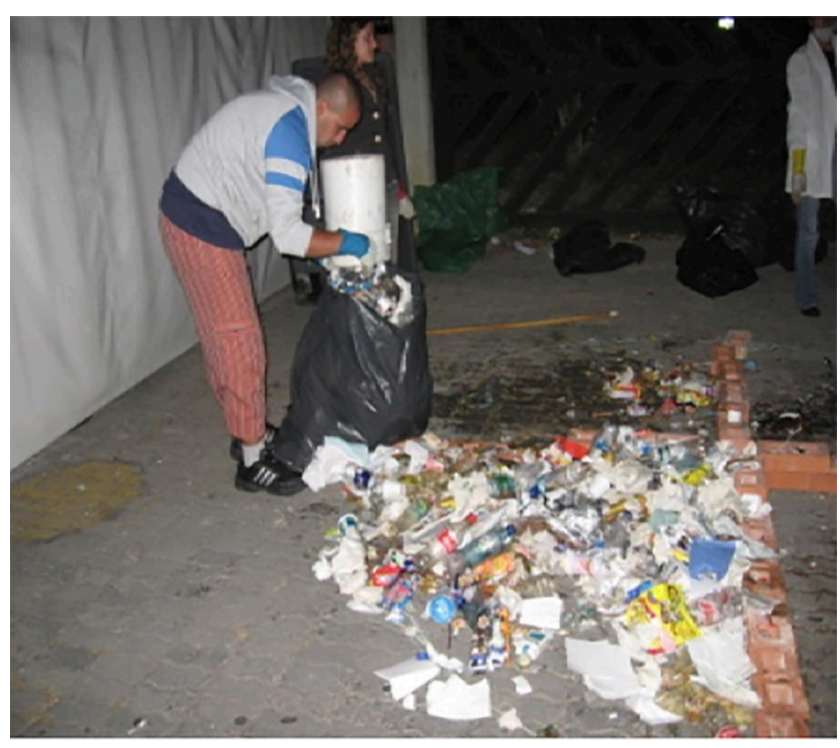

Figura 12. Recogida de los residuos. Fuente: elaboración propia.

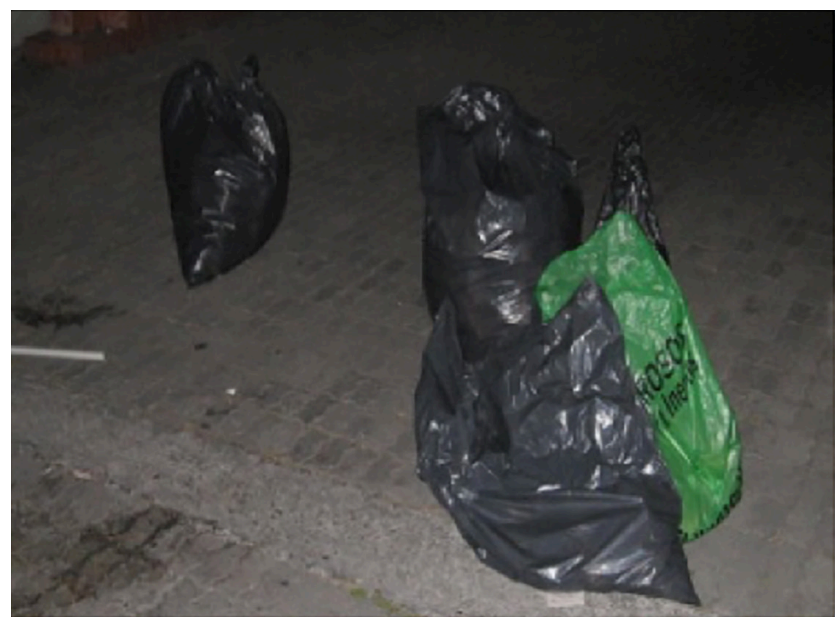

Figura 13. Recogida de las bolsas. Fuente: elaboración propia.

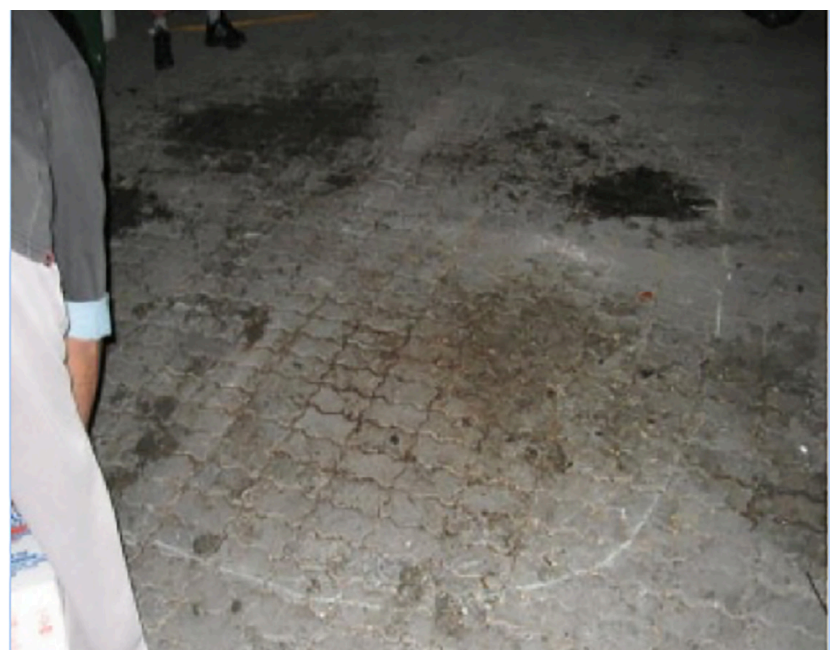

Figura 14. Sitio limpio.

Fuente: elaboración propia.

Para obtener mayor exactitud en el método cuantitativo, la caracterización en cualquier sector de estudio se debe realizar dos, de las tres veces por semana que hace la recolección el vehículo.

Para efectuar este método de cuarteo, se requiere la participación de por lo menos cuatro personas.

Los implementos requeridos son:

- Báscula de piso, con capacidad de $200 \mathrm{~kg}$.

- Bolsas de polietileno de $1.10 \mathrm{~m}$ x $0.90 \mathrm{~m}$ y calibre mínimo del No. 200, para la recolección de cada uno de los materiales que se van a clasificar.

- Guantes gruesos.

- Escobas.

- Tapa bocas. 
El objetivo de la caracterización es conocer la composición y cuantificar los residuos sólidos que se producen durante una semana en un municipio, barrio, institución, empresa o finca.

Para cualquiera de los sitios mencionados anteriormente, el método de caracterización mediante el cuarteo que se utiliza es el mismo, lo que cambia es la manera de tomar la muestra dependiendo del origen, por lo cual se explicara con algunos casos de estudio.

\section{CASOS DE ESTUDIO}

\section{A. Caso de estudio para una finca o empresa}

Para una finca o empresa, en este caso la finca El Franco en Garagoa Boyacá, la cuantificación de residuos se realiza con la toma de la muestra de residuos sólidos generados por día durante una semana, y luego se procede a realizar la caracterización por medio del cuarteo para la determinación de su composición física. Esta consiste en recolectar la muestra de un día, se colocan los residuos sobre un lugar plano y pavimentado; posteriormente se forma un círculo, se homogeniza la muestra y se sigue la metodología planteada para llevar a cabo el cuarteo.

Para la obtención de los cálculos se efectúa el promedio por tipo de residuo con la siguiente fórmula:

\section{Ecuación 1. Promedio por tipo de residuo}

$\begin{gathered}\text { Promedio por } \\ \text { tipo de residuo }\end{gathered}=\frac{K g \text { cuadrante I }+K g \text { cuadrante III }}{2}$

Luego se procede a calcular el porcentaje de cada componente:

\section{Ecuación 2. Porcentaje por tipo de residuo}

$$
\text { Porcentaje }(\%)=\frac{K g \text { por tipo de residuo }}{\begin{array}{c}
K g \text { promedio de residuos } \\
\text { recolectados }
\end{array}} * 100
$$

Finalmente se calcula los kilogramos por semana, de acuerdo con el tipo de residuo:

\section{Ecuación 3. Producción semanal \\ Producción $=(\%$ tipo de residuo $) * \begin{gathered}(\text { Kg total de } \\ \text { residuos por semana })\end{gathered}$}

La tabla I y la figura 15 muestran la caracterización de los residuos sólidos con el uso del desarrollo de las ecuaciones anteriores. Se encuentra, como era de esperarse en este sector, presencia de residuos peligrosos (Respel).

Para el cuarteo es preciso señalar que los cuadrantes I y III tuvieron una diferencia de 1,7 kg ver "tabla I", lo cual está dentro del margen de diferencia propuesto por el método.

\begin{tabular}{|l|c|c|c|c|c|}
\hline \multicolumn{1}{|c|}{ Componente } & $\begin{array}{c}\text { Cuadrante I } \\
(\mathbf{K g})\end{array}$ & $\begin{array}{c}\text { Cuadrante III } \\
(\mathbf{K g})\end{array}$ & $\begin{array}{c}\text { Promedio } \\
(\mathbf{k g})\end{array}$ & $\begin{array}{c}\text { Porcentaje } \\
(\%)\end{array}$ & $\begin{array}{c}\text { Producción } \\
(\mathbf{K g} / \mathbf{s e m})\end{array}$ \\
\hline Residuos de cosecha & 17,1 & 14,2 & $(\mathrm{~kg})$ & Porcentaje & 66,01 \\
\hline Papel & 0,2 & 0,5 & $(\%)$ & Producción & 1,48 \\
\hline Plástico & 0,3 & 0,6 & $(\mathrm{Kg} / \mathrm{sem})$ & 2,04 & 1,90 \\
\hline Vidrio & 3,2 & 2,1 & 2,65 & 12,02 & 11,18 \\
\hline Papel higiénico & 0,5 & 0,1 & 0,3 & 1,36 & 1,27 \\
\hline Latas de hojalata & 0,6 & 0,4 & 0,5 & 2,27 & 2,11 \\
\hline Respel & 1 & 3,3 & 2,15 & 9,75 & 9,07 \\
\hline Total & 22,9 & 21,2 & 22,05 & 100,00 & 93 \\
\hline
\end{tabular}

Tabla I. Caracterización de residuos sólidos finca El Franco Fuente: elaboración propia. 


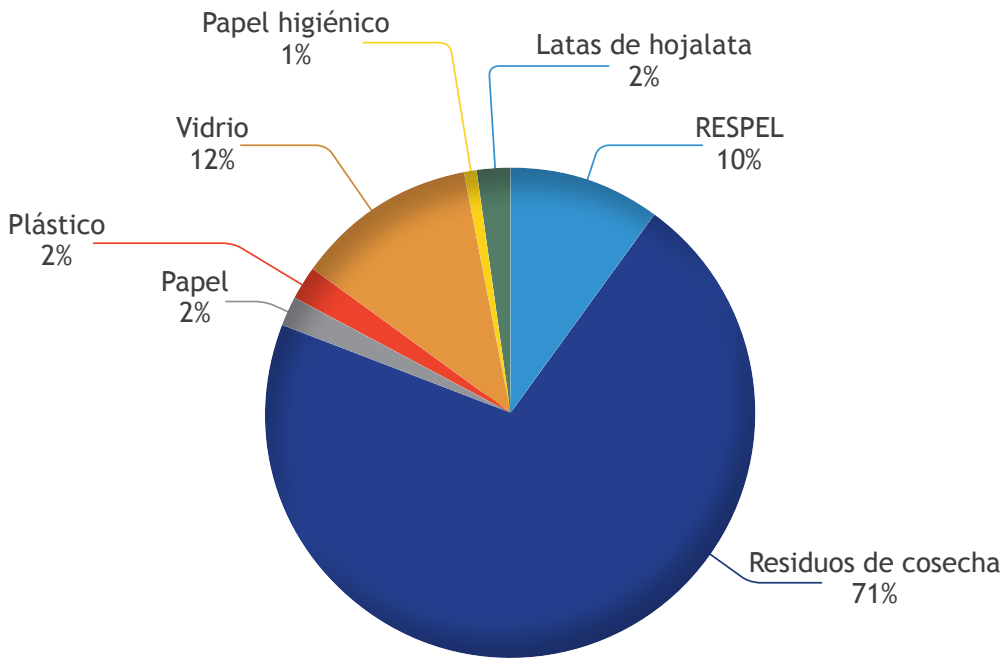

Figura 15. Caracterización de residuos sólidos Finca El Franco. Fuente: elaboración propia.

\begin{tabular}{|c|c|c|c|}
\hline No. & Día & $\begin{array}{r}\text { Residuos } \\
\text { Sólidos }(\mathrm{Kg} / \mathrm{dia})\end{array}$ & Observaciones \\
\hline 1 & Martes & 27,2 & Finalización total de la cosecha de arveja. \\
\hline 2 & Miércoles & 3,2 & Preparación del terreno \\
\hline 3 & Jueves & 18,6 & Hubo cosecha de maíz \\
\hline 4 & Viernes & 15,3 & Reunión familiar \\
\hline 5 & Sábado & 25 & Cáscara de arveja y residuos de mazorca \\
\hline 6 & Domingo & 1,2 & Comercialización \\
\hline 7 & Lunes & 2,6 & Preparación del terreno \\
\hline Total $(\mathrm{Kg} / \mathrm{sem})$ & & 93,1 & \\
\hline
\end{tabular}

Tabla II. Cuantificación de residuos sólidos en la Finca El Franco. Fuente: elaboración propia.

En la tabla II, se presenta la cuantificación diaria en $\mathrm{Kg}$, lo mismo que la fuente generadora de residuos en la finca. Para conocer la cantidad de estiércol producido de la actividad ganadera, se realizó la cuantificación de peso por día del estiércol de los 11 bovinos, los cuales se aislaron durante 1 semana en una hectárea para facilitar la recolección de todo lo generado por el ganado. (Tabla III).

\begin{tabular}{|c|c|}
\hline Día & Estiércol (Kg/día) \\
\hline Martes & 141,7 \\
\hline Miércoles & 139,4 \\
\hline Jueves & 142,5 \\
\hline Viernes & 139,8 \\
\hline Sábado & 141,2 \\
\hline Domingo & 143,4 \\
\hline Lunes & 140,2 \\
\hline Total (Kg/sem) & 998,2 \\
\hline
\end{tabular}

Tabla III. Producción de estiércol del ganado. Fuente: elaboración propia. 
Por consiguiente, la producción total de residuos sólidos para la finca El Franco es de 1081,3 Kg/sem., como se observa en la tabla IV.

\section{Producción de residuos sólidos (Kg/sem)}

\begin{tabular}{|c|c|}
\hline Estiércol & 998,2 \\
\hline Residuos sólidos & 93,1 \\
\hline Total & $\mathbf{1 0 8 1 , 3}$ \\
\hline
\end{tabular}

Tabla IV. Total de residuos sólidos en la finca El Franco Fuente: elaboración propia.

\section{B. Caso de estudio para un municipio}

Para este caso de estudio se toma el municipio de Anolaima, cuya condición para que la caracterización se lleve a cabo correctamente, es que la muestra que se tome contenga todos los materiales que se puedan presentar, para lo cual, el día del muestreo, dentro del camión de recolección no se puede hacer ningún tipo de separación previa. La muestra de $90 \mathrm{Kg}$ como mínimo, debe ser tomada directamente del camión de recolección y paleada de diferentes puntos del mismo, directamente al suelo, para realizar el cuarteo, siguiendo el método aquí planteado.

\begin{tabular}{|l|c|c|c|c|c|}
\hline \multicolumn{1}{|c|}{ Componente } & Cuadrante I & $\begin{array}{c}\text { Cuadrante III } \\
(\mathbf{K g})\end{array}$ & $\begin{array}{c}\text { Promedio } \\
(\mathbf{k g})\end{array}$ & $\begin{array}{c}\text { Porcentaje } \\
(\mathbf{\%})\end{array}$ & $\begin{array}{c}\text { Producción } \\
(\mathbf{K g} / \mathbf{s e m})\end{array}$ \\
\hline Residuos de comida & 12,45 & 16,40 & 14,425 & 62,47 & 14,99 \\
\hline Papel & 1,16 & 0,93 & 1,045 & 4,53 & 1,09 \\
\hline Catón & 0,56 & 0,20 & 0,38 & 1,646 & 0,39 \\
\hline Plástico & 2,45 & 2,20 & 2,325 & 10,07 & 2,42 \\
\hline Textiles & 0,39 & 0,33 & 0,36 & 1,56 & 0,37 \\
\hline Cuero & 0,47 & 0,18 & 0,325 & 1,41 & 0,34 \\
\hline Vidrio & 0,77 & 0,64 & 0,705 & 3,05 & 0,73 \\
\hline Latas & 2,45 & 2,10 & 2,275 & 9,85 & 2,36 \\
\hline Suciedad & 1,40 & 1,10 & 1,25 & 5,41 & 1,30 \\
\hline Total & 22,10 & 24,08 & 23,09 & 100,0 & 24,00 \\
\hline
\end{tabular}

Tabla V. Caracterización de residuos sólidos en el municipio de Anolaima Fuente: elaboración propia.

El municipio tiene una producción de 24 toneladas semanales de residuos sólidos como se observa en la tabla V, de los cuales el $62,47 \%$ o sea 14,99 toneladas por semana son de residuos de comida (figura 16), un porcentaje un poco alto, comparado con otros municipios en los que el porcentaje de residuos de comida se encuentra entre el $55 \%$ y el $60 \%$, y esto es debido a que Anolaima es la capital frutera de Colombia. 


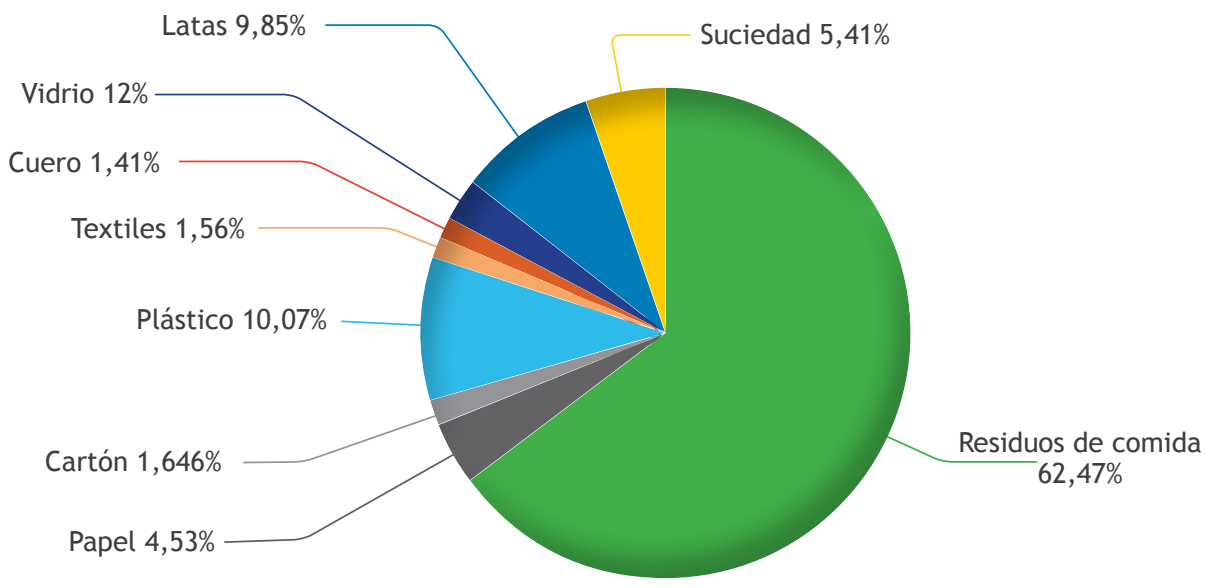

Figura 16. Caracterización de residuos sólidos en el municipio de Anolaima. Fuente: elaboración propia.

\section{Caso de estudio para el barrio Las Villas, Bogotá}

La ciudad de Bogotá cuenta con un total de 1922 barrios, agrupados en Unidades de Planeamiento Zonal (UPZ), que a su vez componen 20 localidades.

- Identificación del barrio: el barrio Las Villas se encuentra ubicado en la UPZ Niza, de la localidad de Suba [6] (figura 17), entre la avenida carrera Suba y la carrera 58, y entre las calles 127 y 129 , como se puede observar en el mapa de la figura 18 [7].

\section{Barrios por UPZ localidad $\mathrm{n}^{\circ} 11$ de suba}

24 Niza

Las Villas

Fig. 17. Barrios de la UPZ Niza. Fuente: [6].

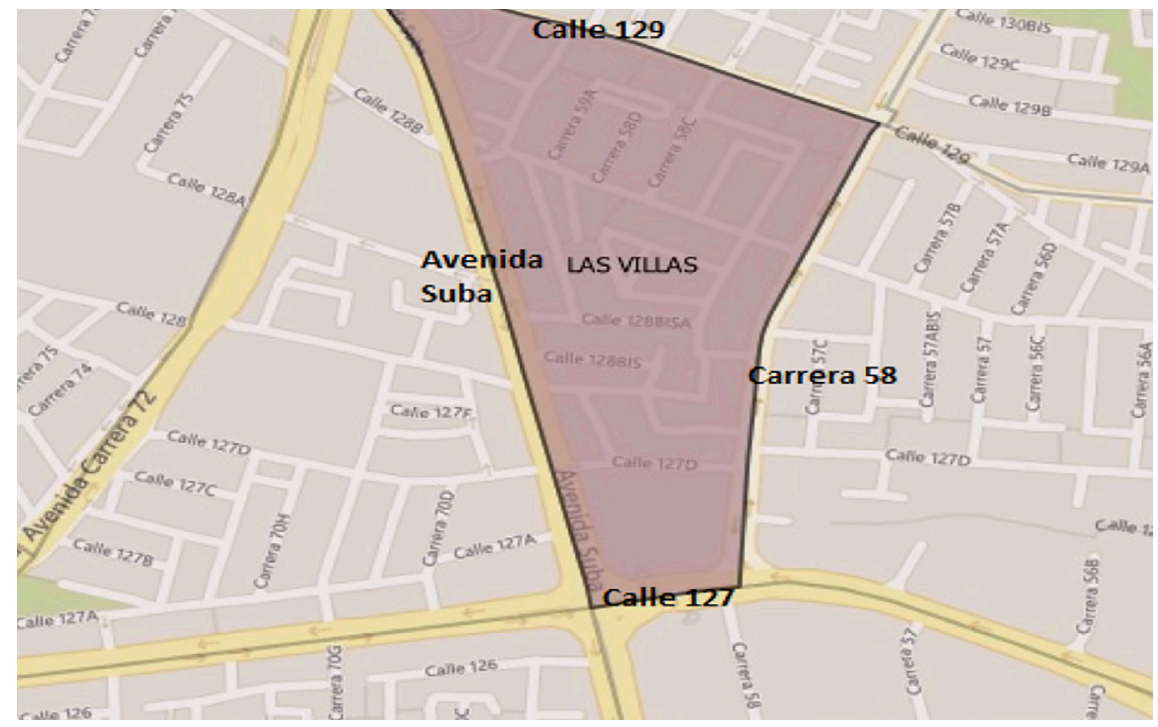

Fig. 18. Barrio Las Villas con límites.

Fuente: [7]. 
El barrio está catalogado dentro del estrato 4 (figura 19) [8]. En su interior se encuentra el Centro Comercial Boulevard Niza (entre carreras avenida Suba y 58, y calles 127 y 127D). El barrio se considera residencial principalmente, sin embargo, se puede observar que como en todos los barrios de Bogotá, ya está involucrada la parte comercial, en esencia restaurantes, bares, farmacias y otros pequeños comercios (fuera del centro comercial Boulevard Niza).

\section{Estratificación socioeconómica urbana}

Localidad No. 11 - Suba - Decreto 394 de julio 29 de 2017
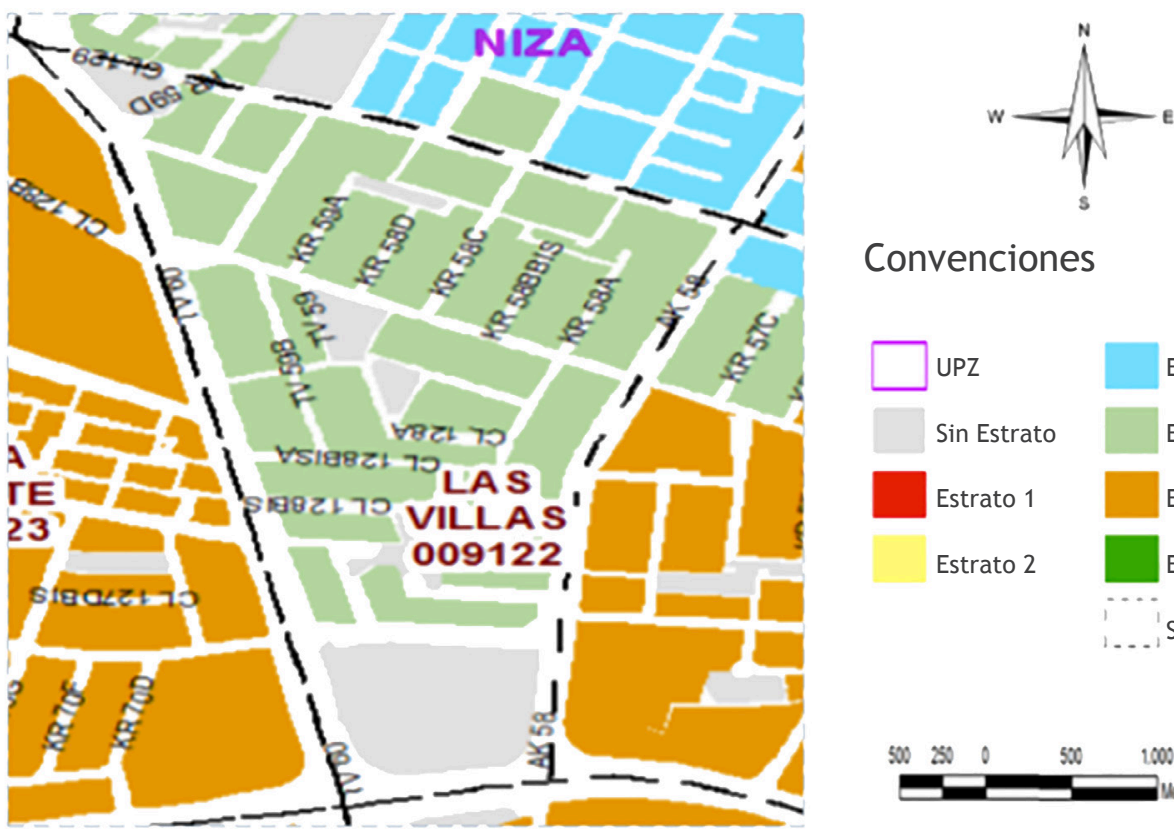

\section{Convenciones}
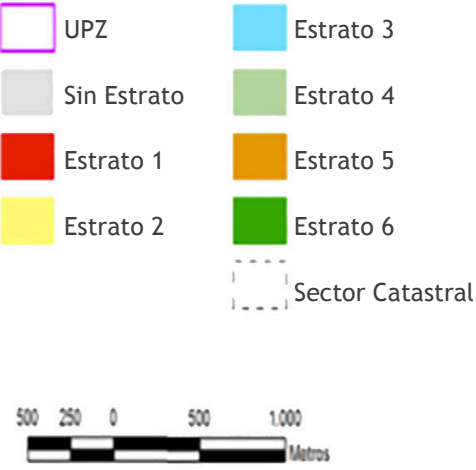

Fig. 19. Estrato barrio Las villas.

Fuente: [8]

Toma de muestra: para el caso de un barrio, la muestra se toma directamente en el origen y no de una carga mezclada de residuos de un vehículo recolector.

Por consiguiente, se deben tomar las bolsas de residuos en los diferentes puntos de generación seleccionados previamente, como casas, edificios, restaurantes, bares, entre otros (figura 20) y llevados al sitio donde se va a llevar a cabo el cuarteo (figura 21). Así se garantiza que la muestra contiene todos los tipos de materiales que se generan allí.

Se pesan como mínimo los $90 \mathrm{Kg}$ y se procede a realizar el cuarteo (figura 22).

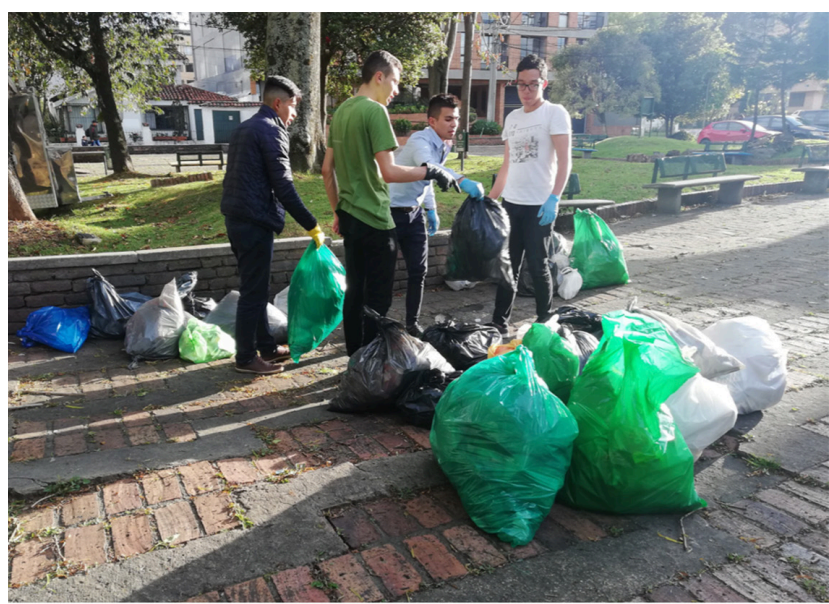

Fig. 20. Recolección de bolsas de diferentes puntos del barrio. Fuente: elaboración de un estudiante. 


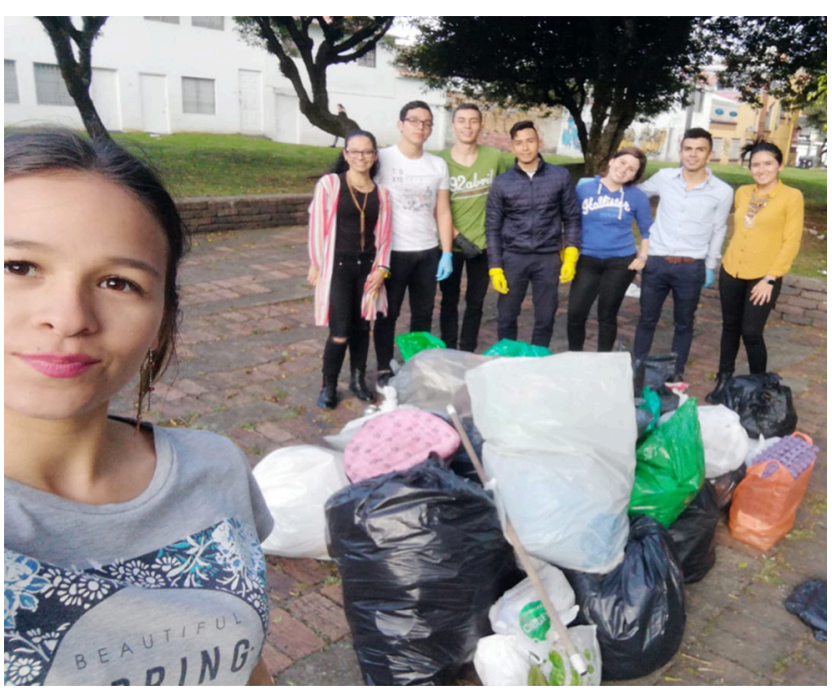

Fig. 21. Localización del sitio.

Fuente: elaboración de un estudiante.

- Caracterización: con los valores obtenidos de cadacuadrantesedeterminael promedioenpesoy el porcentaje, de acuerdo con las ecuaciones 1 y 2. En las tablas VI y VII se muestran los resultados correspondientes.

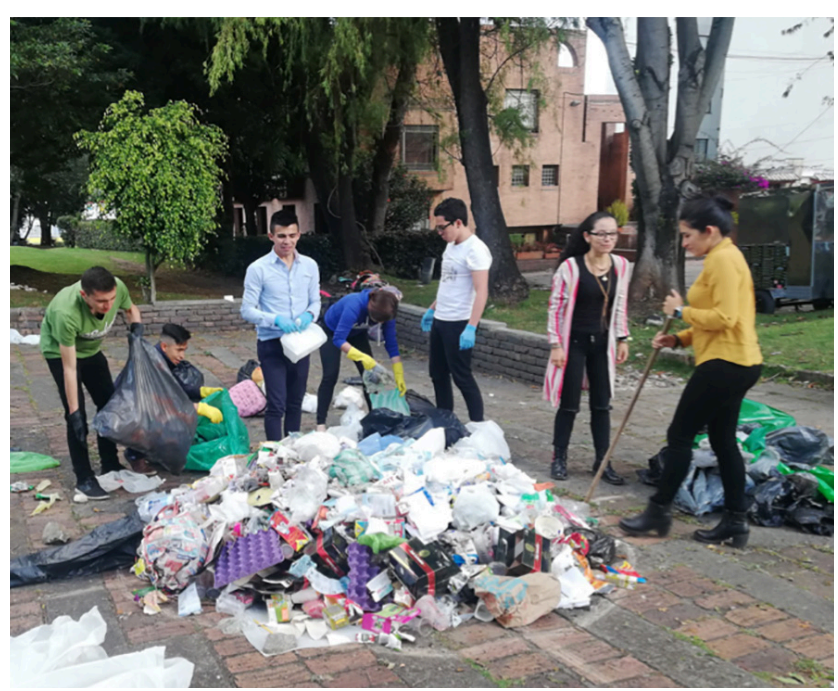

Fig. 22. Realización de cuarteo.

Fuente: elaboración de un estudiante.

En la figura 23 se observan los porcentajes obtenidos para cada componente dentro de la caracterización del barrio Las Villas.

\begin{tabular}{|l|c|c|c|c|c|}
\hline \multicolumn{1}{|c|}{ Aprovechable } & $\begin{array}{c}\text { Cuadrante I } \\
(\mathbf{K g})\end{array}$ & $\begin{array}{c}\text { Cuadrante III } \\
(\mathbf{K g})\end{array}$ & $\begin{array}{c}\text { Promedio } \\
(\mathbf{k g})\end{array}$ & $\begin{array}{c}\text { Porcentaje } \\
(\%)\end{array}$ & T/semana \\
\hline Papel & 1 & 1,5 & 1,25 & 5,9 & 1,59 \\
\hline Cartón & 1,5 & 0,75 & 1,125 & 5,3 & 1,43 \\
\hline Plástico & 3,0 & 3,75 & 3,375 & 15,9 & 4,29 \\
\hline Textiles & 0,5 & 1 & 0,75 & 3,5 & 0.95 \\
\hline Goma & 0,1 & 0 & 0,05 & 0,2 & 0,06 \\
\hline Cuero & 0,3 & 0,2 & 0,25 & 1,2 & 0,32 \\
\hline Vidrio & 1,5 & 2,25 & 1,875 & 8,8 & 2,39 \\
\hline Latas & 0,1 & 0,2 & 0,15 & 0,7 & 0,19 \\
\hline Aluminio & 0,2 & 0,1 & 0,15 & 0,7 & 0,19 \\
\hline No aprovechables & 12,5 & 12 & 12,25 & 57,7 & 15,58 \\
\hline Total & 20,7 & 21,75 & 21,225 & 100 & 27 \\
\hline
\end{tabular}

Tabla VI. Caracterización de residuos barrio las villas y expresión en toneladas por semana Fuente: elaboración propia. 


\section{- Cálculos}

\begin{tabular}{l|c|c|c|}
\hline \multicolumn{1}{|c|}{ Componente } & Promedio & Porcentaje & Toneladas/Semana \\
\hline Papel & $(1,0+1,5) / 2=1,25 \mathrm{~kg}$ & $1,25 / 21,225 \times 100=5,9 \%$ & $0,059 \times 27=1,59$ \\
\hline Cartón & $(1,5+0,75) / 2=1,125 \mathrm{~kg}$ & $1,125 / 21,225 \times 100=5,3 \%$ & $0,053 \times 27=1,43$ \\
\hline Plásticos & $(3,0+3,75) / 2=3,375 \mathrm{~kg}$ & $3,375 / 21,225 \times 100=15,9 \%$ & $0,159 \times 27=4,29$ \\
\hline Textiles & $(0,5+1,0) / 2=0,75 \mathrm{~kg}$ & $0,75 / 21,225 \times 100=3,5 \%$ & $0,035 \times 27=0,95$ \\
\hline Goma & $(0,1+0) / 2=0,05 \mathrm{~kg}$ & $0,05 / 21,225 \times 100=0,2 \%$ & $0,002 \times 27=0,06$ \\
\hline Cuero & $(0,3+0,2) / 2=0,25 \mathrm{~kg}$ & $0,25 / 21,225 \times 100=1,2 \%$ & $0,012 \times 27=0,32$ \\
\hline Vidrio & $(1,5+2,25) / 2=1,875 \mathrm{~kg}$ & $1,875 / 21,225 \times 100=8,8 \%$ & $0,088 \times 27=2,39$ \\
\hline Latas & $(0,1+0,2) / 2=0,15 \mathrm{~kg}$ & $0,15 / 21,225 \times 100=0,7 \%$ & $0,007 \times 27=0,19$ \\
\hline Aluminio & $(0,2+0,1) / 2=0,15 \mathrm{~kg}$ & $0,15 / 21,225 \times 100=0,7 \%$ & $0,007 \times 27=0,19$ \\
\hline No aprovechable & $(12,5+12,0) / 2=12,25 \mathrm{~kg}$ & $12,25 / 21,225 \times 100=57,7 \%$ & $0,577 \times 27=15,58$ \\
\hline
\end{tabular}

Tabla VII. Cálculos de promedio, porcentaje y toneladas totales por semana Fuente: elaboración propia.

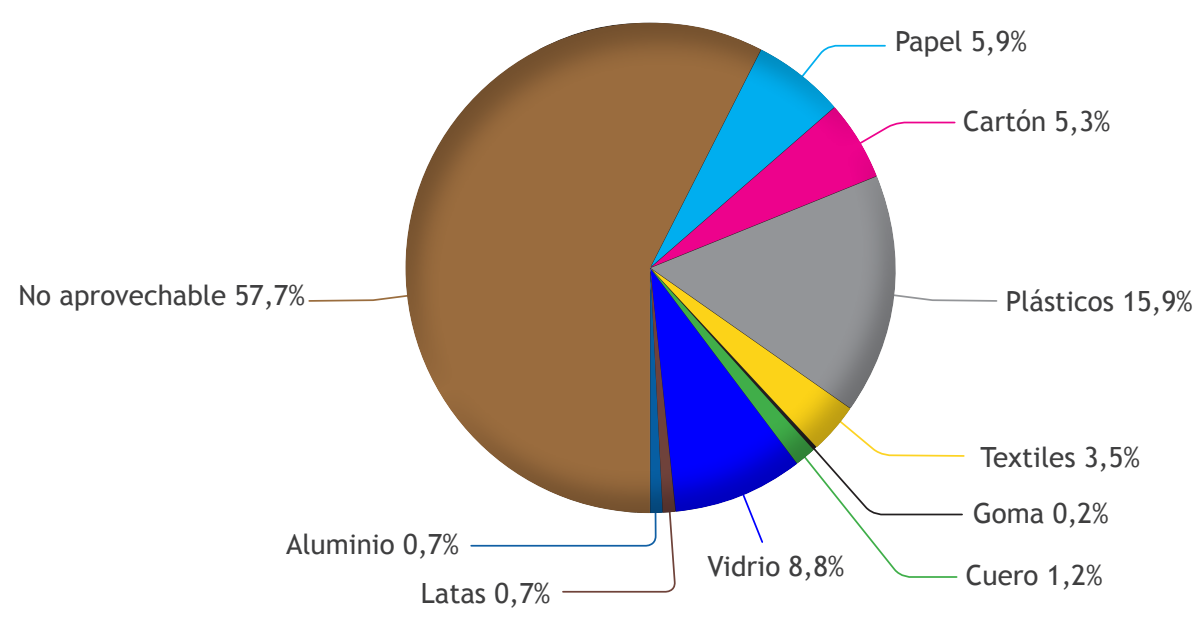

Figura 23. Caracterización de residuos sólidos barrio Las Villas.

Fuente: elaboración propia.

- Producción: la información de la producción fue obtenida mediante entrevista al señor Enrique Pamplona [9], conductor del camión recolector de placas ESM 754 y con número 5156 de la empresa Área Limpia, figura 24, que es la encargada de la prestación del servicio de aseo en el barrio Las Villas de Bogotá; información verificada telefónicamente con la empresa.

El recorrido se realiza los martes, jueves y sábados, entre las 7 y 9 de la noche, son los martes los días de mayor cantidad de residuos recogidos con 11 toneladas, los jueves y sábados 8 toneladas. La producción total entonces es de 27 toneladas semanales, que no incluyen el centro comercial Boulevard Niza.

De la cantidad total, mediante la caracterización, se obtiene la producción por cada uno de componentes encontrados y esto es lo que se presenta en la última casilla de las tablas VI y VII. 

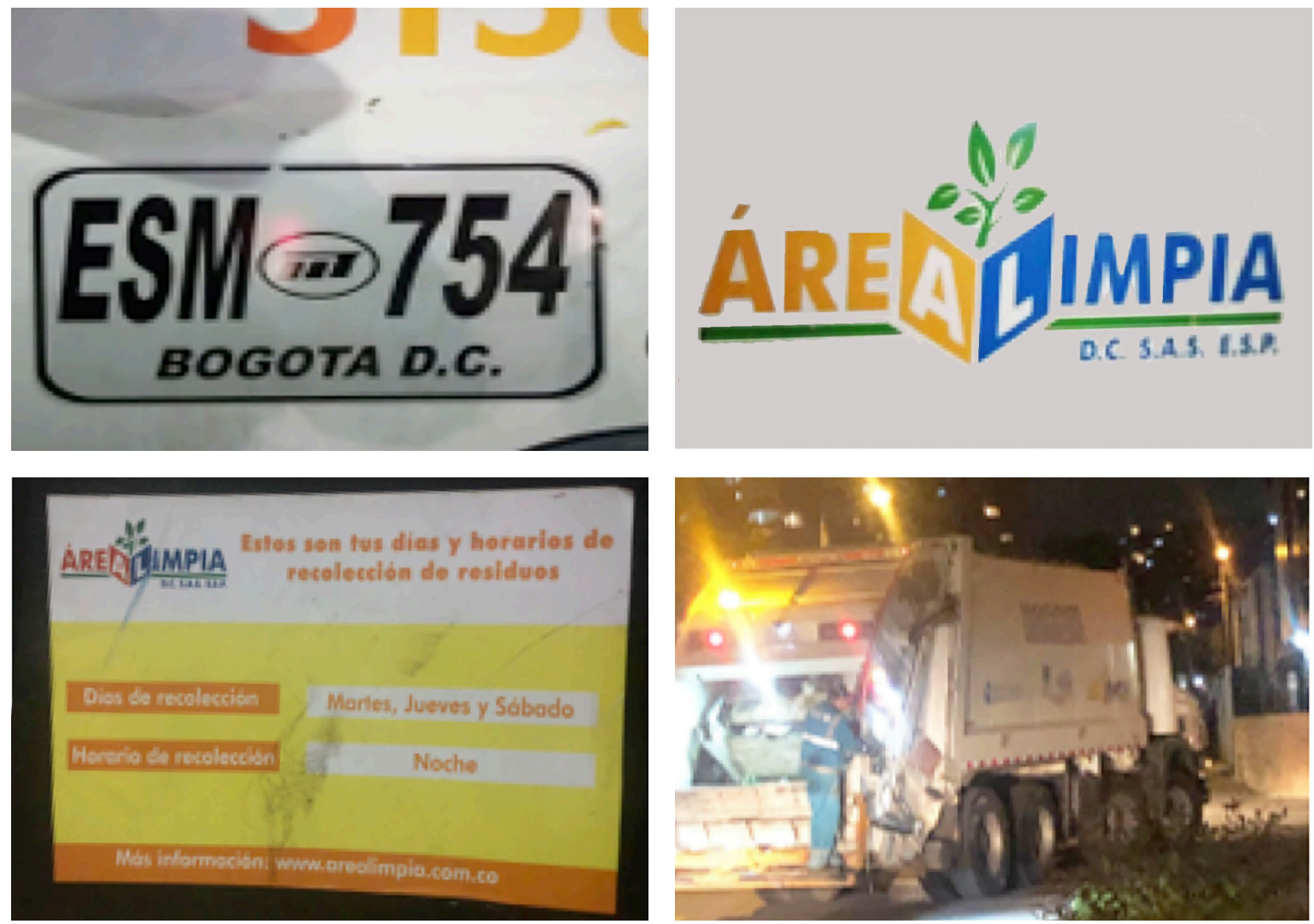

Fig. 24. Características del camión compactador en el barrio Las Villas. Placa, número, empresa y horario de recolección. Fuente: elaboración propia.

- Población: de acuerdo con el censo 2005 del Departamento Administrativo Nacional de Estadística (DANE), la población del barrio Las Villas es de 2698 habitantes y se requiere conocer la población del barrio para el año 2019, año en que se lleva a cabo la caracterización.

Según el análisis estadístico del Geoportal del DANE [10], y a partir del censo de 2005, se realizó una proyección de la población para el barrio, basada en la tasa de crecimiento de la UPZ Niza, que se obtuvo del "Visor de Proyecciones" elaborada por la Secretaría de Planeación, específicamente por la Subsecretaría de Información y Estudios Estratégicos [11], como se observa en la secuencia a continuación:

Se traza el área sobre el Geoportal, y se obtiente la población de 2005 para el barrio Las Villas, de 2698 personas. (figura 25). 


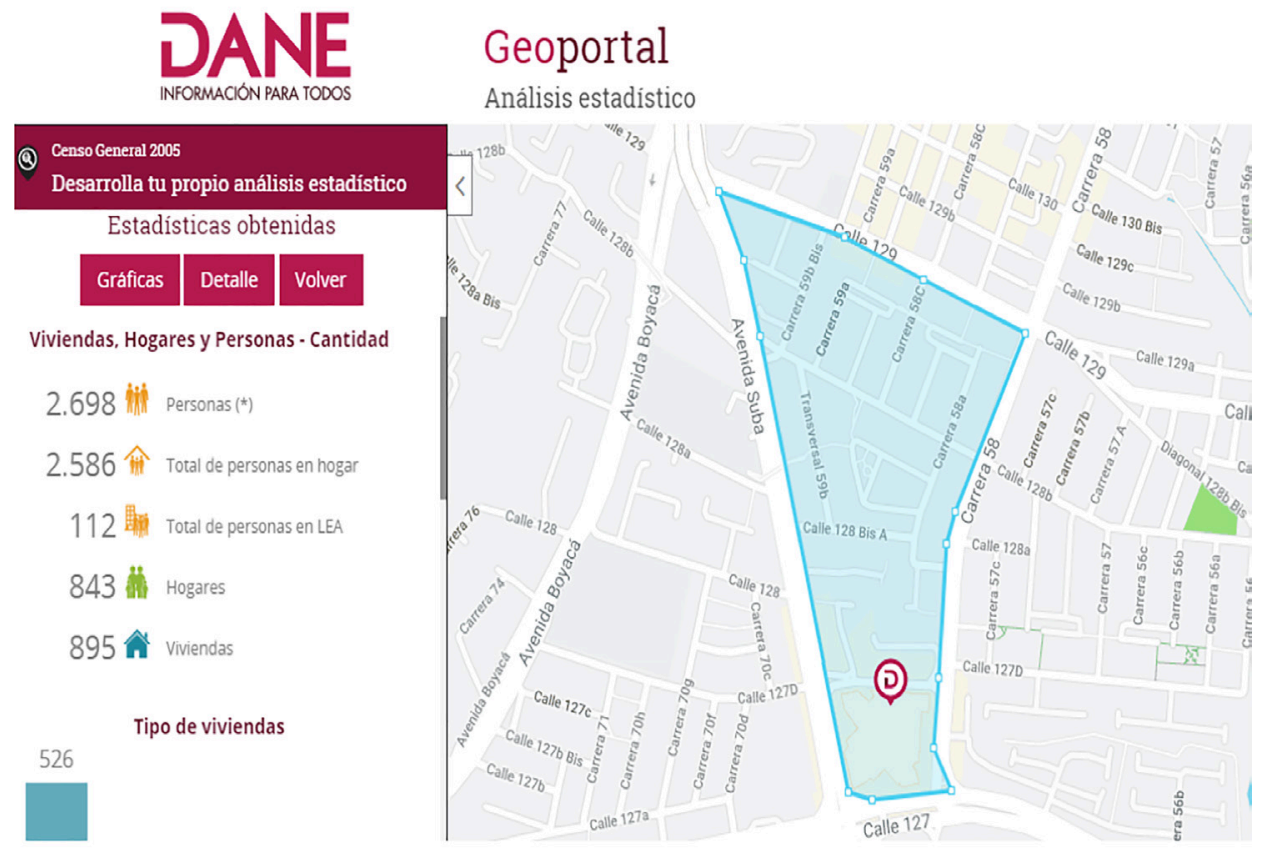

Fig. 25. Mapa Geoportal DANE (2019), población del barrio Las Villas en 2005. Fuente: [10].

A continuación, desde la página de la Secretaría de Planeación - Home - Gestión estudios estratégicos - Estudios macro - Proyecciones de población - Descargar. (figura 26) [11].

Dentro del documento, se ingresa a "Estructura UPZ". (figura 27).

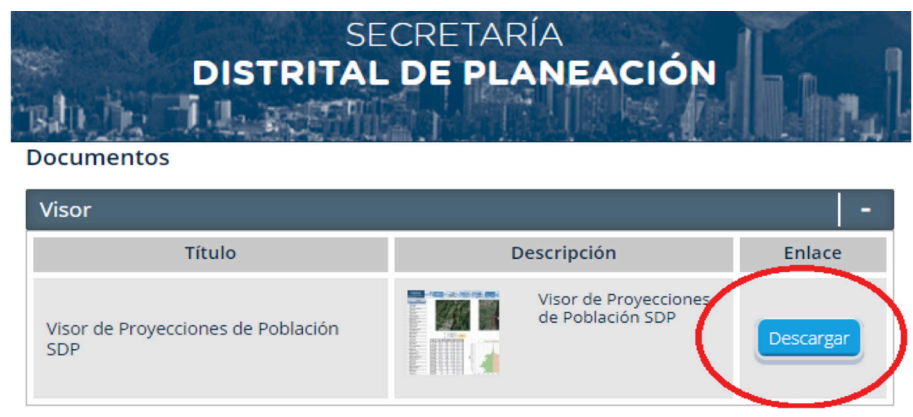

Fig. 26. Página de acceso al visor de proyecciones de la Secretaría de Planeación. Fuente: [11].

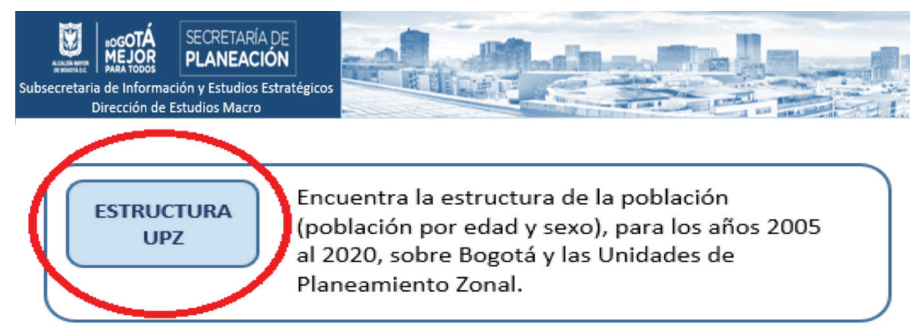

Fig. 27. Menú del “Visor de Proyecciones” de la Secretaría de Planeación. Fuente: [11]. 
Allí, en la lista de UPZ, se encuentra "Niza", dentro de la localidad 11 de Suba, en la que aparece una gráfica de población proyectada hasta 2030, y de la cual se obtiene, la tasa de crecimiento anual. (figura 28).

\section{Barrios por UPZ localidad $n^{\circ} 11$ de suba}

24 Niza

Las Villas

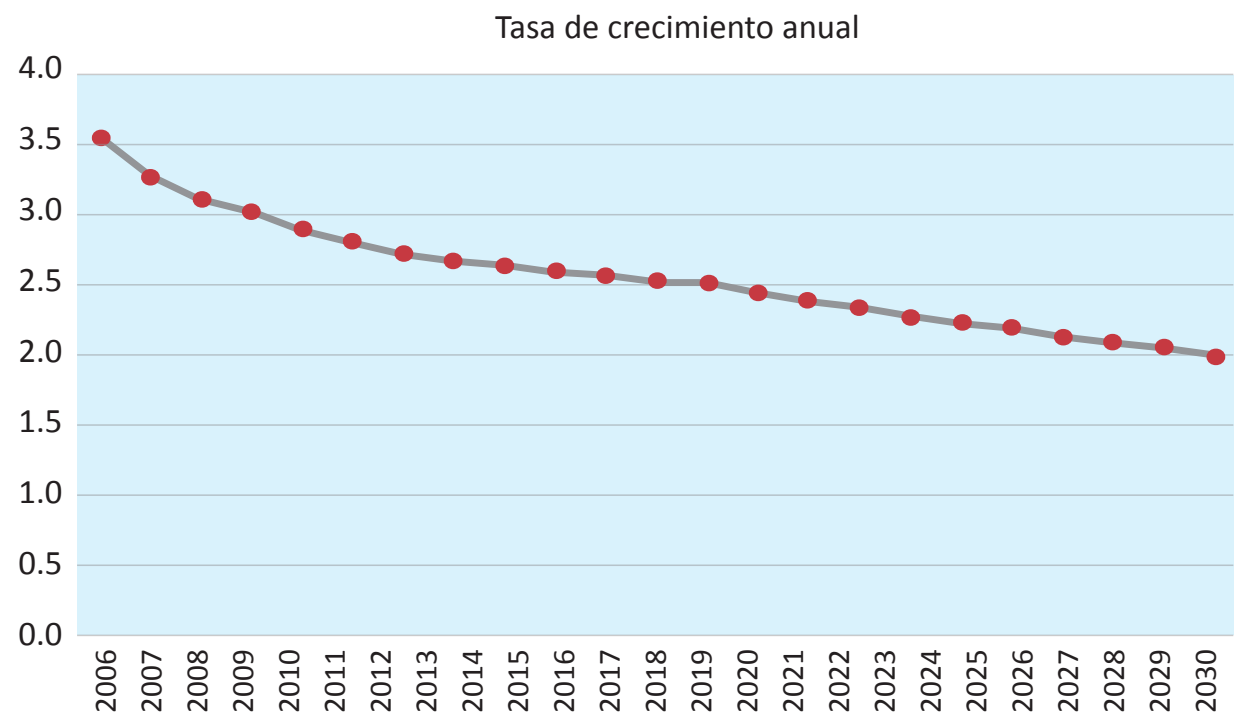

Año

Fig. 28. Gráfica de proporción de crecimiento poblacional en la UPZ Niza.

Fuente: [11].

Para cada año, existe una tasa de crecimiento individual para la UPZ, que se aplica para calcular la población anual del barrio Las Villas, de manera que, asumiendo cierta homogeneidad y partiendo de los 2698 habitantes censados en 2005, se llega a una proyección de 3971 habitantes en el barrio Las Villas, para 2019. (Tabla VIII).

Tabla VIII. Proyección anual de la población del barrio Las Villas hasta el año 2019 Fuente: elaboración propia.

\begin{tabular}{|c|c|}
\hline Año & Población (hab) \\
\hline 2005 & 2698 \\
\hline 2006 & 2790 \\
\hline 2007 & 2882 \\
\hline 2008 & 2971 \\
\hline 2009 & 3060 \\
\hline 2010 & 3149 \\
\hline 2011 & 3237 \\
\hline 2012 & 3325 \\
\hline 2013 & 3414 \\
\hline 2014 & 3503 \\
\hline 2015 & 3594 \\
\hline 2016 & 3688 \\
\hline 2017 & 3780 \\
\hline 2018 & 3874 \\
\hline 2019 & 3971 \\
\hline
\end{tabular}


- Producción per cápita: con los datos de producción se calcula la producción per cápita del barrio, así:

\section{Ecuación 4. Producción per cápita}

$$
p p c=\frac{27 \frac{\text { ton }}{\mathrm{sem}}\left(\frac{1000 \mathrm{~kg}}{1 \mathrm{ton}}\right)\left(\frac{1 \mathrm{sem}}{7 \text { días }}\right)}{3971 \mathrm{hab}}=0,97 \frac{\mathrm{kg}}{\text { día } \mathrm{hab}}
$$

De acuerdo con la Alcaldía Mayor de Bogotá en su Decreto 548 de 2015, por el cual se adopta el Plan de Gestión Integral de Residuos Sólidos (PGIRS), del Distrito Capital, y se dictan otras disposiciones. [12], se tiene que el promedio de la producción per cápita en el año 2019 para Bogotá es de 0,9296 kg/ hb.día. (Tabla IX).

Bogotá tiene seis estratos socioeconómicos, lo que significa que el promedio equivale a un estrato 3. Por consiguiente, el resultado obtenido para el barrio Las Villas de $0,97 \mathrm{~kg} / \mathrm{hb}$.dia, es correcto para el estrato 4 en el 2019, año en el que fue realizado el trabajo.

\begin{tabular}{|c|c|c|c|c|}
\hline Año & Habitantes & $\begin{array}{c}\text { Generación } \\
\text { (Ton/dia) }\end{array}$ & $\begin{array}{c}\text { PPC } \\
(\mathbf{k g} / \\
\text { hb.dia) }\end{array}$ & $\begin{array}{c}\text { Aumento } \\
(\%)\end{array}$ \\
\hline 2016 & 7.977 .000 & 7275,87 & 0,9121 & 0,67 \\
\hline 2017 & 8.080 .000 & 7419,52 & 0,9183 & 0,68 \\
\hline 2018 & 8.184 .000 & 7566,00 & 0,9245 & 0,55 \\
\hline 2019 & 8.283 .000 & 7699,80 & 0,9296 & 0,55 \\
\hline 2020 & 8.383 .000 & 7835,97 & 0,9347 & 0,56 \\
\hline 2021 & 8.484 .000 & 7974,54 & 0,9400 & 0,55 \\
\hline
\end{tabular}

Tabla IX. Decreto 548 de 2015. Alcaldía mayor de Bogotá Fuente: elaboración propia.

Este resultado en el que la ppc obtenida por cálculos coincide con la ppc del estrato real en el que se esté trabajando, para este caso el estrato del barrio Las Villas, confirma que la cuantificación estuvo bien realizada y por consiguiente la caracterización también es correcta.

\section{CONCLUSIONES}

Esta guía cumple con el propósito de dar a conocer un método sencillo y eficaz, comparado con los escasos métodos que se han propuesto para determinar la caracterización y cuantificación de residuos sólidos.

El método presentado en esta guía permite caracterizar diferentes sectores como el municipal, urbano, institucional, entre otros, como se puede observar en los casos de estudio, lo que cambia para cada sector es la forma de realizar el muestreo dependiendo del origen de los residuos sólidos.

La diferencia entre los métodos propuestos y el presentado en esta guía es del $10 \%$, un valor relativamente pequeño, comparado con la facilidad para realizarlo, y por consiguiente un ahorro de tiempo, personal e implementos.

Este método arroja datos congruentes entre la ppc calculada y la real del sitio donde se realiza el cuarteo, lo que indica que la composición y la caracterización son correctas.

\section{REFERENCIAS}

[1] G. Tchobanoglous, Gestión integral de residuos sólidos. España, McGrawHill, 1994.

[2] M. Cabeza Díaz, "Riesgo climático y definición de estrategias financieras para su mitigación en el sector agua y saneamiento en ALC: Residuos sólidos: Visión de la gestión de los desechos sólidos ante el cambio climático", Banco Interamericando de Desarrollo, 2020. [En línea]. Disponible en: http://dx.doi. org/10.18235/0002751. [Accedido: 30agosto-2020].

[3] Organización Panamericana de la Salud, "OPS/CEPIS/04/IT-634 Anexo 2 guía para caracterización de residuos sólidos domiciliarios". [En línea]. Disponible en: https: / / docplayer.es/20690705-Ops-cepis04-it-634-original-espanol-pagina-59-anexo2-guia-para-caracterizacion-de-residuossolidos-domiciliarios.html. [Accedido: 12marzo-2020]. 
[4] K. Sakurai, Plan de Gestión ambiental de los residuos sólidos urbanos de la ciudad de Reque, 1982. [En línea]. Disponible en: sidalc.net. [Accedido: 20-enero-2020].

[5] Secretaría de Desarrollo Urbano y Ecología, "NMX-AA-015-1985 Protección al ambiente - contaminación del suelo - residuos sólidos municipales - muestreo - método de cuarteo", 1985. [En línea]. Disponible en: http:// legismex.mty.itesm.mx/normas/aa/aa015. pdf. [Accedido: 15-febrero-2020].

[6] R. y D. Secretaría Distrital de Cultura, "Listado de barrios por UPZ", 2019. [En línea]. Disponible en: https://www. culturarecreacionydeporte.gov.co/sites/ default/files/convocatorias_cartillas_y_ anexos/listado_de_barrios_46.pdf. [Accedido: 15-febrero-2020].

[7] Ideca, "Barrio las Villas Sector Catastral, Bogotá D.C.”, 2020. [En línea]. Disponible en: https: / / mapas. bogota.gov.co/ ?l=654\&e =74.17375562306833,4.5682225959272875,74.00896070119356,4.651123497981-397, 4686\&b=7256. [Accedido: 08-abril-2020].
[8] Secretaría Distrital de Planeación, Estratificación Socioeconómica Urbana de la Localidad No. 11 Suba Decreto 394, 2017. [En línea].

[9] E. Pamplona, Interviewee, Entrevista a conductor de camión recolector, Empresa Área Limpia. [Entrevista]. 2019.

[10] DANE, "Geoportal DANE información para todos", [En línea]. Disponible en: https: / geo portal.dane.gov.co/. [Accedido: 21-abril-2020].

[11] Secretaría Distrital de Planeación, "Proyecciones de población". [En línea]. Disponible en: http://www.sdp.gov.co/gestion-estudiosestrategicos/estudios-macro/proyeccio nes-de-poblacion. [Accedido: 19-diciembre-2019].

[12] Alcaldía Mayor deBogotá D.C., “Decreto548de 18 de diciembre de 2015. Por el cual se adopta el Plan de Gestión Integral de Residuos Sólidos, PGIRS, del Distrito Capital, y se dictan otras disposiciones", 2015. [En línea]. Disponible en: http://legal.legis.com.co/document/ Index?obra=legcol\&document=legcol_ e0c2a987d2f74be39559ef98869aefde. [Accedido: 10-mayo-2020]. 